\title{
Valoración funcional en artroplastía total de rodilla comparando la preservación del ligamento cruzado posterior versus posteroestabilización
}

\author{
Functional assessment in total knee arthroplasty comparing the posterior \\ cruciate ligament preservation versus postero-stabilization
}

\author{
Chico-Carpizo F, ${ }^{*}$ Domínguez-Gasca LG, ${ }^{\ddagger}$ Orozco-Villaseñor SL*
}

Hospital General de León.

RESUMEN. Introducción: Los dos tipos de prótesis que más se utilizan en la actualidad son la prótesis total de rodilla con preservación del ligamento cruzado posterior y con sustitución del ligamento cruzado posterior. Objetivo: Valorar si existe diferencia en la funcionalidad entre ambos procedimientos. Material y métodos: Estudio observacional, retrospectivo y transversal con 134 pacientes divididos en grupo $A(N=67)$ con preservación del ligamento y grupo B ( $\mathrm{N}=67)$ con posteroestabilización. Uso de la Escala de la Asociación Americana para Prótesis de Rodilla con la modificación de Insall para valorar funcionalidad. Análisis estadístico: Uso de $\chi^{2}$ con corrección de Yates. Resultados: En el grupo A los resultados: excelentes (31\%); buenos (24\%); regulares (22\%) y malos (22\%). En el grupo B los resultados: excelentes (34\%); buenos (31\%); regulares (16\%) y malos (19\%); sin diferencia estadísticamente entre ambos grupos. Conclusiones: No se observó diferencia estadísticamente significativa entre edad, género, lateralidad de la lesión ni entre número de pacientes, con resultados excelentes, buenos regulares y malos de acuerdo con la escala utilizada. Llama la atención el elevado porcentaje de malos resultados en ambos grupos de esta serie en la prótesis de rodilla.

Palabras clave: Prótesis de rodilla, ligamento cruzado posterior, sustitución, preservación resultados.
ABSTRACT. Introduction: The two most commonly used types of prostheses are the total knee prosthesis with posterior cruciate ligament preservation and posterior cruciate ligament replacement. Objective: To assess whether there is a difference in functionality between the two procedures. Material and methods: Observational, retrospective and cross-sectional study with 134 patients divided into group $A(\mathrm{~N}=67)$ with ligament preservation and group $\mathrm{B}(\mathrm{N}=67)$ with posterior substituting. Using the American Association Scale for Knee Prosthetics with Insall modification to assess functionality. Statistical analysis: use of $\chi^{2}$ with Yates correction. Results: In group A the results: excellent (31\%); good (24\%); regular (22\%) bad (22\%). In group B the results: excellent (34\%); good (31\%); regular (16\%) and bad (19\%); without statistical difference between the two groups. Conclusions: No statistically significant difference was observed between age, gender, laterality of injury, or between number of patients with excellent results, good regular and bad according to the scale used. The high percentage of poor results in both groups of this series for the knee prosthesis is striking.

Keywords: Knee prosthesis, posterior cruciate ligament, replacement, preservation results.

\section{Nivel de evidencia: IV}

* Ortopedista. Cirugía Articular. División de Cirugía del Hospital General de León. León, Guanajuato, México.

‡ Ortopedista. Cirugía Articular. División de Cirugía del Hospital Ángeles León. León, Guanajuato, México.

Correspondencia:

Dr. Fernando Chico-Carpizo

E-mail: fernandochico1089@gmail.com

Recibido: 20-06-2020. Aceptado: 10-02-2021.

Citar como: Chico-Carpizo F, Domínguez-Gasca LG, Orozco-Villaseñor SL. Valoración funcional en artroplastía total de rodilla comparando la preservación del ligamento cruzado posterior versus posteroestabilización. Acta Ortop Mex. 2021; 35(1): 69-74. https://dx.doi.org/10.35366/100934 


\section{Introducción}

La osteoartrosis (OA) se define como una enfermedad articular crónica degenerativa, caracterizada por un componente inflamatorio acompañado de degeneración, pérdida progresiva de cartílago hialino y hueso subcondral así como daño del tejido sinovial, engrosamiento y esclerosis de la lámina subcondral, formación de osteofitos, distensión de la cápsula articular y cambios en los tejidos blandos periarticulares. ${ }^{1}$ Es una de las principales causas de dolor musculoesquelético y discapacidad a nivel mundial en pacientes adultos con una prevalencia superior a $44.7 \%$.

Su prevalencia en mayores de 45 años oscila de 7 a $19 \%$ en mujeres y de 6 a 13\% en hombres con un riesgo mayor en el género femenino. La prevalencia de OA ha aumentado en todo el mundo, lo que implica la pérdida aproximada de 2.3 años de vida saludable. En México es una de las primeras 10 causas de invalidez total, lo que incrementa la carga económica y social del país. Se espera que su frecuencia siga elevándose debido al incremento de la esperanza de vida y a los hábitos poco saludables de la población.

La artroplastía total de rodilla (ATR) consiste en el cambio de las superficies articulares dañadas de la rodilla. En los Estados Unidos se realizan un millón de artroplastías totales de rodilla y de cadera/año. ${ }^{3,4}$ Con el envejecimiento de la población, aumento en el diagnóstico y tratamiento de estadios avanzados de la osteoartritis, los procedimientos de recambio articular tienen proyectado convertirse en la cirugía electiva más común en los Estados Unidos en las próximas décadas, ${ }^{5}$ con proyección de aumento de $600 \%$ en 2030. De ahí la importancia de la correcta reconstrucción de la rodilla para prevenir posibles complicaciones. ${ }^{6}$

Los dos tipos de prótesis que más se utilizan en la actualidad son la prótesis total de rodilla con preservación del ligamento cruzado posterior y la posteroestabilizada o con sustitución del ligamento cruzado posterior (Figura 1). Se presenta un estudio comparativo, descriptivo y multicéntrico que valora los resultados funcionales entre pacientes que utilizan la retención o sustitución del ligamento cruzado posterior en nuestro medio.

\section{Material y métodos}

Con protocolo aprobado por los comités de ética de ambas instituciones y cumpliendo las normas vigentes para la investigación en salud mexicana y la declaración de Helsinki, considerándolo como de riesgo mínimo de acuerdo con el Título Quinto de la Ley General de Salud, se realizó un estudio observacional, retrospectivo y transversal en pacientes postoperados de artroplastía total de rodilla para el tratamiento de osteoartrosis de los años 2014 a 2018 en el Hospital General León y en el Hospital Regional de Alta Especialidad del Bajío que cumplieron con los criterios de inclusión: a) paciente postoperado de artroplastía total primaria de rodilla entre Enero de 2014 y Diciembre de 2018 en alguna de las dos instituciones mencionadas, que acudieron a valoración después de al menos seis meses del postoperatorio; b) aceptación del paciente a participar en el estudio y firma de consentimiento informado; c) con capacidad intelectual de responder a la evaluación; d) paciente que acuda a la consulta de valoración para realizar estudio. Los criterios de exclusión fueron: a) pacientes con antecedentes de cirugías agregadas en extremidad ipsilateral a artroplastía de rodilla; b) alteración funcional o en rangos de movilidad en extremidad operada, por causas diferentes a la artroplastía total de rodilla (neurológicas, circulatorias, cardiovasculares, metabólicas); c) no aceptar participar en el estudio; d) ausencia a consulta de valoración postoperatoria; e) paciente sometido a artroplastía de
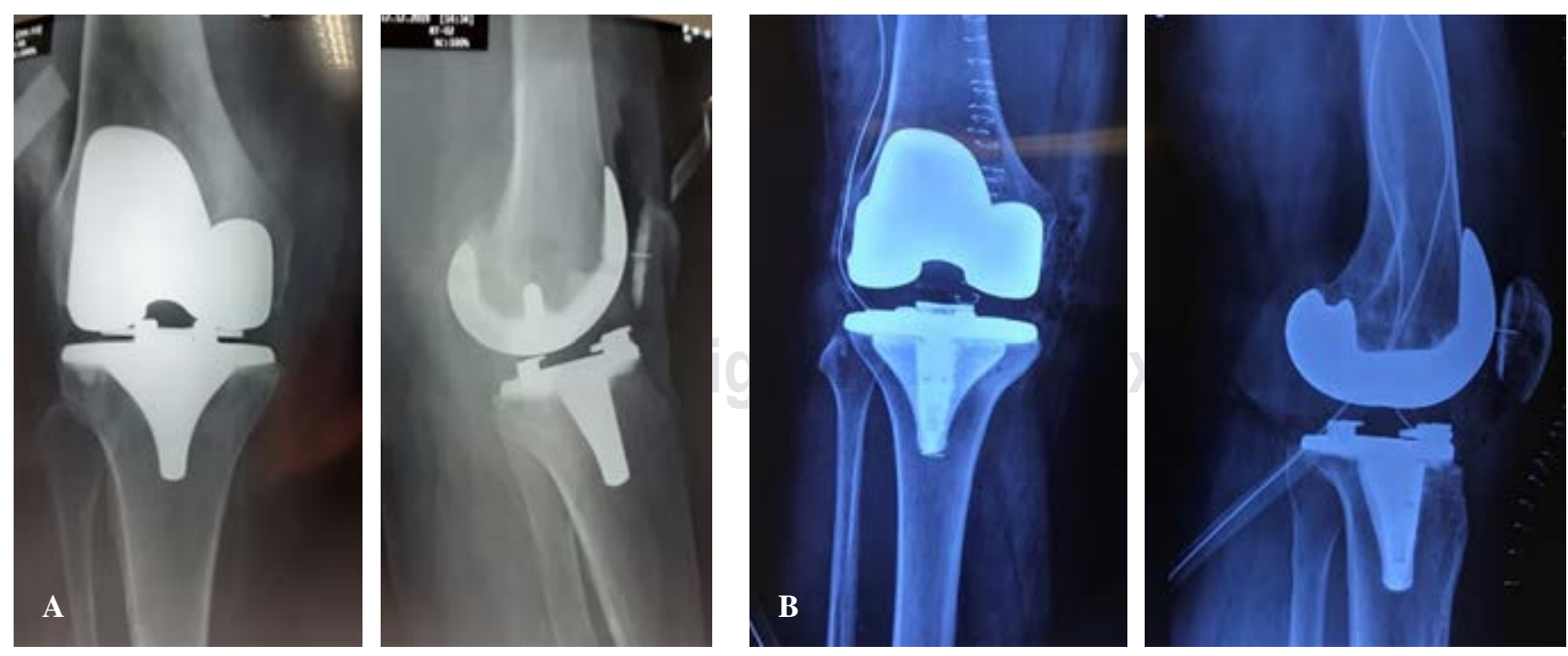

Figura 1: Radiografías anteroposterior y lateral de pacientes con artroplastía total de rodilla. A) Preservación del ligamento cruzado posterior. B) Posteroestabilización. 
revisión de rodilla; f) complicación posterior a la artroplastía total de rodilla.

El estudio incluyó las siguientes variables: edad, género, lateralidad, además se efectuó valoración de funcionalidad con la Escala de la Asociación Americana para Prótesis de Rodilla ${ }^{7}$ (EAAPR) con la modificación de Insall y colaboradores $^{8}$ con validación en 2016 por Thomsen ${ }^{9}$ y su grupo, corroborando resultados con los obtenidos en la dirección electrónica oficial de la Knee Society of Orthopedics (http:// www.orthopaedicscore.com/scorepages/knee_society_score.html), la cual está conformada de dos partes; la primera consta de valoración de datos clínicos y de exploración, mientras que la segunda valora funcionalidad; calificando los datos obtenidos como excelentes con puntaje $\geq 80$; buenos de 70 a 79 puntos; regulares de 60 a 69 y malos menores de 60 puntos.

Se realizó análisis estadístico con el programa SPSS 21 para Windows, representando como frecuencia y porcentaje la comparación entre grupos por prueba de $\chi^{2}$ con corrección de Yates dado que la muestra fue menor de 200 individuos; considerando el valor de $\mathrm{p}=0.05$ como estadísticamente significativo.

\section{Resultados}

El universo estudiado comprendió 134 pacientes, de éstos 67 correspondieron al Hospital Regional de Alta Especialidad del Bajío, postoperados de prótesis total de rodilla con preservación del ligamento cruzado posterior (denominado grupo A) y 85 al Hospital General León, postoperados de prótesis total de rodilla posteroestabilizada; del último grupo se evaluaron 67 pacientes (denominado grupo B), ya que 18 no cumplieron con los criterios de inclusión.

El grupo A: artroplastía total de rodilla con retención del ligamento cruzado posterior. (R)

Conformado por 67 pacientes presentó edad promedio \pm desviación estándar (DE) de 70 años \pm 13.61 y recorrido de 47 a 87 años, su distribución por género mostró $68.6 \%$ $(n=46)$ en el sexo femenino y $31.3 \%(n=21)$ en el masculino, correspondiendo la relación de género 2:1 a favor del femenino $(\mathrm{p}=0.03)$ sin diferencia estadísticamente significativa.

Los resultados de la escala de valoración se muestran en la figura 2.

La asociación entre edad y los resultados de la escala EAAPR mostró ausencia de significancia estadística en las dos partes constituyentes de la misma.

La relación de género y los resultados de EAAPR mostró diferencia entre la primera parte de la escala con respecto a resultados malos, siendo mayores $17.4 \%(\mathrm{n}=8)$ en el género femenino, mientras que en el masculino los resultados malos se observaron en 9.5\% $(n=2)(p=0.05)$; la relación de género con la segunda parte de la escala

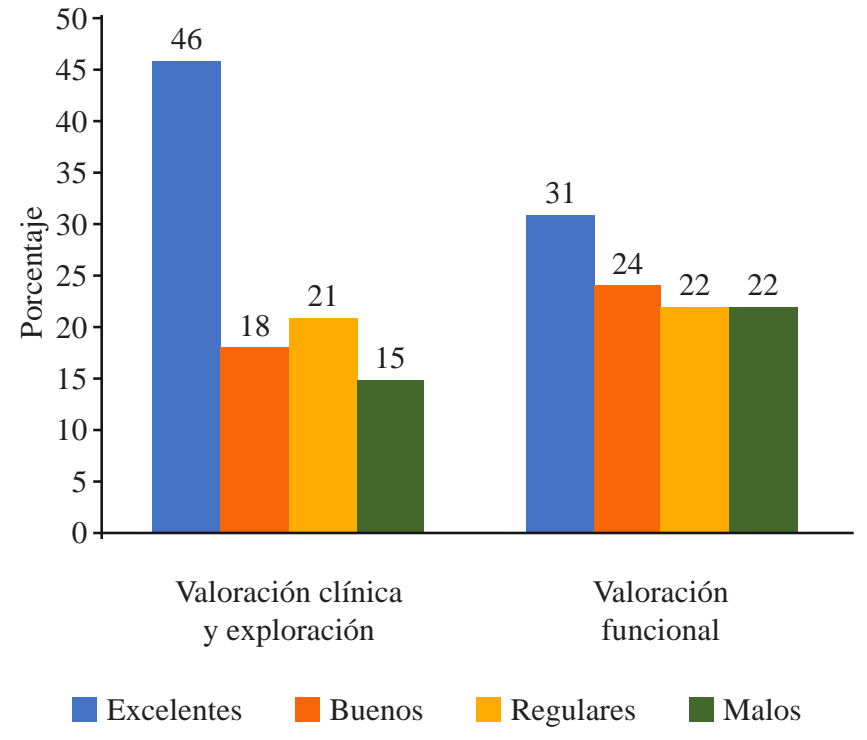

Figura 2: Resultados de valoración en la Escala de la Asociación Americana para Prótesis de Rodilla en 67 pacientes con retención del ligamento cruzado posterior.

EAAPR mostró un franco predominio de resultados excelentes y buenos en el género masculino (sumando más de $75 \%$ ), por otra parte en el femenino la suma de resultados regulares y malos correspondió a $55 \%$ sin significancia estadística $(\mathrm{p}=0.74)$.

La distribución por lado operado fue semejante: izquierda $50.75 \%(n=34)$ y derecha $49.25 \%(n=33) \sin$ diferencia estadísticamente significativa, no influyendo en resultados de la escala EAAPR en ninguna de sus partes ( $\mathrm{p}$ $=0.525)$ en la primera parte de la escala y $(p=0.73)$ en la segunda.

\section{El grupo B: artroplastía total de rodilla con sustitución del ligamento cruzado posterior. (S)}

Conformado por 67 pacientes valorados en la consulta con edad promedio $\pm \mathrm{DE}$ correspondiendo a $68.4 \pm 12$ años con recorrido de 48 a 88 años. La asociación entre edad y los resultados de la escala EAAPR mostró ausencia de significancia estadística en ambas partes constituyentes de la misma.

La distribución por género fue de 67.16\% ( $\mathrm{n}=45)$ en el sexo femenino y $32.84 \%(n=22)$ en el masculino. Correspondiendo la relación de género 2:1 a favor del femenino $(\mathrm{p}=0.03)$.

La distribución por lado fue $56.71 \%(\mathrm{n}=38)$ en el izquierdo y $43.28 \%(n=29)$ en el derecho; en $17.91 \%(n=$ 12) bilaterales. La distribución por lado operado fue semejante, sin diferencia estadísticamente significativa, no influyendo en resultados de la escala EAAPR en ninguna de las dos partes.

En la figura 3 se muestran los resultados de la evaluación de la EAAPR. 


\section{Discusión}

En este estudio, tanto en el grupo A como en el B no se observó diferencia significativa en relación con la lateralidad; respecto al género existió una relación 2:1 a favor del femenino, lo que implica que la rodilla femenina por ser de menor tamaño y con masa muscular de menor volumen que la del hombre, además del valgo acentuado por las características de la pelvis, la puede hacer más susceptible de daño articular; por otra parte se anota que los resultados funcionales excelentes fueron mayores en el género masculino, con mayor número en el grupo $\mathrm{B}$.

En relación con la escala EAAPR se debe mencionar que la primera parte de la misma, la cual valora datos clínicos y de exploración, no necesariamente correlaciona con la segunda parte de la escala que valora funcionalidad, pues se pueden encontrar datos de la primera parte excelentes, con resultados de funcionalidad regulares y malos y a la inversa, por lo que deben existir otros factores que influyen en la funcionalidad como pueden ser: el estado anímico, la capacidad cognitiva, la actitud del paciente, la condición física, el tiempo de evolución de la osteoartritis antes de cirugía, la presencia de comorbilidades, el sedentarismo originado por dolor articular, la presencia de contracturas en flexión de caderas y rodillas, fibrosis articular, proceso inflamatorio persistente así como constancia y calidad en los programas de rehabilitación prequirúrgicos y postquirúrgicos, el manejo de tejidos blandos durante la cirugía, el tamaño del inserto de polietileno y el tamaño del componente patelar, el umbral al dolor, el apoyo intrafamiliar, por último, la presencia de complicaciones como sinovitis, fibrosis e incluso trombosis venosa profunda a pesar de manejo adecuado con anticoagulantes. Independientemente de ello, no se

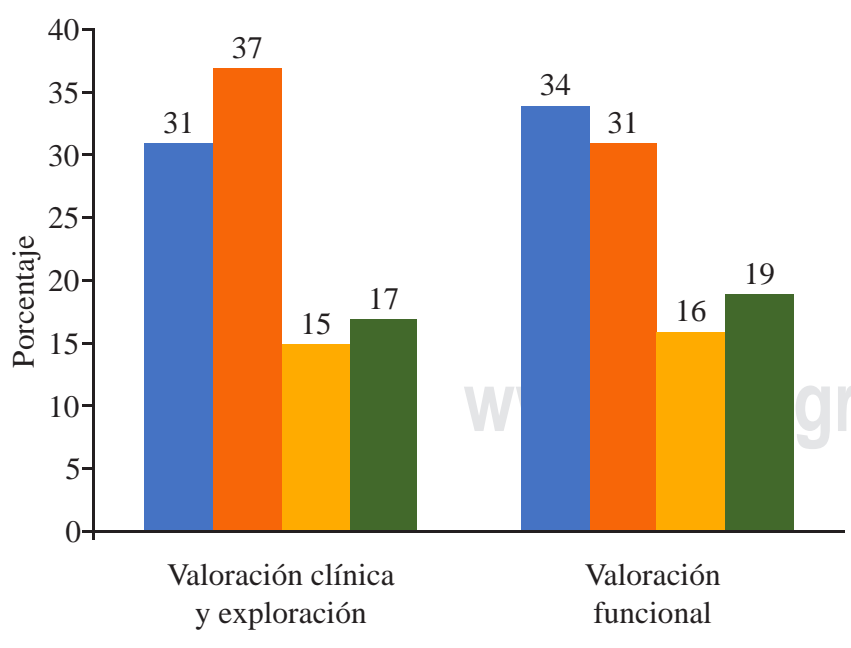

Excelentes Buenos Regulares $\square$ Malos

Figura 3: Resultados de valoración en la Escala de la Asociación Americana para Prótesis de Rodilla en 67 pacientes con prótesis de rodilla posteroestabilizada. encontró diferencia significativa entre los dos grupos al correlacionar los estudios aleatorizados que comparan la retención del ligamento cruzado posterior (LCP) contra la posteroestabilización.

El LCP se considera el principal opositor de la traslación posterior de la tibia y un restrictor secundario del varo, valgo y rotación externa de la rodilla. En los pacientes en quienes se retiene el LCP, éste mantiene la estabilidad en flexión y extensión, brinda diferentes tipos de mecanorreceptores, detectando la posición de la articulación (propiocepción) y movimiento articular (cinestesia). En varios estudios se ha demostrado que al retener el LCP, existe una deficiencia en la traslación femorotibial posterior (roll-back) con la flexión de la rodilla. Esto se debe a un balance inadecuado en flexión del LCP durante la artroplastía. El balanceo del LCP consiste en seleccionar adecuadamente el grosor del inserto y el tamaño de los componentes femoral y tibial, logrando así que el LCP esté tenso en flexión, pero relajado en extensión. Cuando no se realiza un adecuado balanceo del LCP, se logran resultados subóptimos provocando dolor. Si el LCP queda muy laxo, el paciente puede presentar inestabilidad. Si el LCP queda muy tenso el paciente sufre de limitación a la flexión y el inserto de polietileno se somete a un alto estrés provocando desgaste temprano del mismo. Se puede realizar durante el procedimiento una liberación del LCP en pacientes en quienes la flexión es muy limitada. ${ }^{10}$

Cuando se sustituye el LCP utilizando otro diseño protésico, se obtiene aumento del espacio articular o gap en flexión. Este aumento por lo general se compensa con insertos de polietileno más gruesos o componentes femorales más grandes. El diseño de prótesis de rodilla más utilizado cuando se sustituye el LCP es la prótesis total de rodilla posteroestabilizada, este tipo de prótesis tiene un mecanismo de poste para sustituir al LCP, logrando así el roll back femorotibial durante la flexión y mejorar la estabilidad de la rodilla. ${ }^{10}$

Un factor muy discutido a favor y en contra de sacrificar el LCP es el aflojamiento aséptico del componente tibial debido a la presión ejercida por el componente femoral y el polietileno, lo cual lleva a un desgaste prematuro de los componentes provocando fallo temprano de la prótesis, dolor y limitación funcional al paciente. Por otra parte, en las prótesis posteroestabilizadas, la estabilidad en flexión se logra mediante el aumento de la congruencia del polietileno con la superficie del fémur y la colocación del tetón intercondíleo provoca mayor estrés en la interfaz hueso-cemento de la tibia, generando la movilización y aflojamiento protésico precoz. Otra teoría es que ante la conservación del LCP se produce un movimiento de vaivén anteroposterior del fémur sobre la tibia, lo que produce fuerzas alternativas de compresióndistracción que pueden afectar la fijación de la prótesis; sin embargo, no existen estudios clínicos pertinentes que demuestren alguna de estas teorías. Debe mencionarse que el polietileno de una prótesis con retención de cruzado (CR, por sus siglas en inglés) está sometido a mayor 
estrés que el de una posteroestabilizada (PS, por sus siglas en inglés). Esto es debido a la forma que tienen ambos modelos. La superficie articular de una prótesis PS presenta mayor congruencia con la superficie del fémur, lo que hace que exista una mayor superficie de contacto y por lo tanto mayor reparto de la carga. Por el contrario, el polietileno de una prótesis CR es plano, no se adapta a la superficie del fémur, resultando en una menor área de contacto y de esta manera, un incremento del estrés sobre el polietileno, lo que provoca mayor y más temprano desgaste en la prótesis CR.

Se han realizado desde los años 90 estudios aleatorizados que comparan la retención del ligamento cruzado posterior (LCP) contra la posteroestabilización. En 2005 se publicó un metaanálisis de Cochrane en este tema sin poder encontrar diferencias entre los dos tratamientos. Fue imposible dar una recomendación en cuanto a preservar o sacrificar el LCP. En metaanálisis más recientes, como el de Verra ${ }^{10}$ y colaboradores en 2013, tampoco se logró establecer diferencias en cuanto a dolor, rangos de movimiento, resultados clínicos y radiográficos entre las dos técnicas.

En el estudio de Verra ${ }^{11}$ y su equipo en 2015 se encontró una diferencia en cuanto al tiempo de duración de la prótesis, donde grandes cohortes demostraron sobrevivencia de la ATR en 91-90\% de las ATR con preservación del LCP y 76-75\% en las ATR con sustitución del LCP, aunque estos resultados pueden estar influenciados por los diferentes materiales o diseños utilizados en las prótesis.

En 2016 Longo $^{12}$ y colegas realizaron una revisión donde se obtuvieron resultados similares sin diferencia significativa en cuanto a resultados funcionales y complicaciones, con diferencia en cuanto rangos de movilidad, mejores resultados para la ATR con sustitución del LCP y mejoría de la flexión de hasta $4.3^{\circ}$. Sin embargo, otros estudios como el de Chaudar y colaboradores no encontraron diferencia significativa en los rangos de movimiento, de flexión y extensión postquirúrgicos en las dos técnicas, difiriendo con los demás estudios en la literatura.

Estudios biomecánicos recientes demuestran que la insuficiencia del LCP debido a daño previo a la cirugía, sacrificio del LCP o rotura posterior a la ATR se relacionan con traslación femoral anterior, reducción del roll-back femoral, disminución de la fuerza de contacto femorotibial, llevando a una inestabilidad anteroposterior y a un aumento del gap en flexión, sobre todo después de $60^{\circ}$ de flexión. En este estudio se confirma la importancia del LCP durante el balance de tejidos blandos para restringir la traslación tibial posterior, aumentar el roll-back femoral y contribuir a la estabilidad articular, aunque una liberación parcial del LCP puede reducir la tensión del mismo y prevenir un roll-back excesivo que causa un desgaste posterior del componente tibial y el polietileno. ${ }^{13}$

Los factores que influyen en la elección del tipo de prótesis, ya sea posteroestabilizada (sustituye el LCP) o no posteroestabilizada (preserva el LCP), dependen del estado de degenera- ción del ligamento, el tipo de deformidad de la rodilla, el tipo de implante utilizado o la preferencia personal del cirujano. Lombardi ${ }^{14}$ y colaboradores indican una diferencia de $2.4^{\circ} \mathrm{a}$ $3.4^{\circ}$ de flexión y en algunos casos de $9^{\circ}$ a $17^{\circ}$, con diferencia en el roll back de hasta $3 \mathrm{~mm}$, comprobada con radiografías laterales en flexión en las prótesis en las que se realizó sustitución del LCP. ${ }^{10,11,12,13,14,15} \mathrm{Al}$ comparar el dolor, duración del implante, complicaciones y satisfacción del paciente no se encontraron diferencias significativas entre las dos técnicas.

En el presente trabajo existen algunas debilidades como el reducido número de pacientes y el relativo corto tiempo de uso de la prótesis (seis meses a cinco años), lo que pudo haber originado sesgos en los resultados, ya que en ningún caso se detectaron datos de aflojamiento de los componentes protésicos, ni tampoco fue necesaria cirugía de revisión por fallo protésico así como la no valoración y correlación con resultados de los múltiples factores mencionados en renglones anteriores.

\section{Conclusiones}

El presente trabajo correlaciona con la mayoría de los resultados obtenidos en la literatura, no se observaron diferencias estadísticamente significativas en funcionalidad entre la artroplastía total de rodilla con preservación del ligamento cruzado posterior contra la técnica de posteroestabilización, debiendo considerarse la técnica quirúrgica a realizar de manera individual en cada paciente.

Existe un elevado porcentaje en ambos grupos de malos resultados comparados con la literatura internacional.

\section{Referencias}

1. Guía de Práctica Clínica del Instituto Mexicano del Seguro Social. IMSS-726-14. Prevención, diagnóstico y tratamiento de rehabilitación en el paciente adulto con osteoartrosis de rodilla en los tres niveles de atención. México: Secretaría de Salud; 2014.

2. Espinosa MR, Arce SCA, Cajigas MJC, Esquivel VJA, Gutiérrez GJJ, Martínez HJL, et al. Reunión multidisciplinaria de expertos en diagnóstico y tratamiento de pacientes con osteoartritis. Actualización basada en evidencias. Med Int Mex. 2013; 29(1): 67-92.

3. Maradit Kremers H, Larson DR, Crowson CS, Kremers WK, Washington RE, Steiner CA, et al. Prevalence of total hip and knee replacement in the united states. J Bone Joint Surg Am. 2015; 97(17): 1386-97.

4. Steiner C, Andrews R, Barrett M, Weiss A. HCUP projections: mobility/orthopedic procedures 2003 to 2012. 2012. HCUP Projections Report \# 2012-03. 2012 Sep 20. U.S. Agency for Healthcare Research and Quality.

5. Kurtz SM, Lau E, Ong K, Zhao K, Kelly M, Bozic KJ. Future young patient demand for primary and revision joint replacement: national projections from 2010 to 2030. Clin Orthop Relat Res. 2009; 467(10): 2606-12.

6. Tanzer M, Makhdom AM. Preoperative planning in primary total knee arthroplasty. J Am Acad Orthop Surg. 2016; 24(4): 220-30.

7. Ewald FC. The Knee Society total knee arthroplasty roentgenographic evaluation and scoring system. Clin Orthop Relat Res. 1989; (248): 9-12.

8. Insall JN, Dorr LD, Scott RD, Scott WN. Rationale of the Knee Society clinical rating system. Clin Orthop Relat Res. 1989; (248): 13-4.

9. Thomsen MG, Latifi R, Kallemose T, Barfod KW, Husted H, Troelsen A. Good validity and reliability of the forgotten joint score in evaluating the outcome of total knee arthroplasty. Acta Orthop. 2016; 87(3): 280-5. 
10. Verra WC, van den Boom LG, Jacobs W, Clement DJ, Wymenga AA, Nelissen RG. Retention versus sacrifice of the posterior cruciate ligament in total knee arthroplasty for treating osteoarthritis. Cochrane Database Syst Rev. 2013; 2013(10): CD004803.

11. Verra WC, Boom LG, Jacobs WC, Schoones JW, Wymenga AB, Nelissen RG. Similar outcome after retention or sacrifice of the posterior cruciate ligament in total knee arthroplasty. Acta Orthop. 2015; 86(2): 195-201.

12. Longo UG, Ciuffreda M, Mannering N, D'Andrea V, Locher J, Salvatore G, et al. Outcomes of posterior-stabilized compared with cruciate-retaining total knee arthroplasty. J Knee Surg. 2018; 31(4): 321-40. doi: 10.1055/s-0037-1603902.
13. Kebbach M, Grawe R, Geier A, Winter E, Bergschmidt P, Kluess D, et al. Effect of surgical parameters on the biomechanical behaviour of bicondylar total knee endoprostheses - A robot-assisted test method based on a musculoskeletal model. Sci Rep. 2019; 9(1): 14504. doi: 10.1038/s41598-019-50399-3.

14. Lombardi AV Jr, Mallory TH, Fada RA, Hartman JF, Capps SG, Kefauver CA, et al. An algorithm for the posterior cruciate ligament in total knee arthroplasty. Clin Orthop Relat Res. 2001; (392): 75-87.

15. Seon JK, Park JK, Shin YJ, Seo HY, Lee KB, Song EK. Comparisons of kinematics and range of motion in high-flexion total knee arthroplasty: cruciate retaining vs. substituting designs. Knee Surg Sports Traumatol Arthrosc. 2011; 19(12): 2016-22. 